\title{
3D-Printed Patient-Specific Models for CT- and MRI-Guided Procedure Planning
}

mage-guided, minimally invasive percutaneous ablations of primary and metastatic tumors are increasingly being performed in the head/neck and the spine. Thermal (radiofrequency, laser, microwave, cryoablation) and nonthermal (irreversible electroporation) ablation techniques provide symptomatic relief in patients considered to be poor surgical candidates, but are associated with a steep learning curve and often require careful planning given the individual patient's anatomy. ${ }^{1}$ Safety is also a concern, ${ }^{2}$ particularly as new application sites are explored, such as the posterior spinal elements. ${ }^{3}$

$3 \mathrm{D}$ printing is an emerging technology for presurgical planning and simulation, intraoperative navigation, and physician training. ${ }^{4}$ Models of patient anatomy that are 3D-printed from medical images provide spatial comprehension and tactile feed-
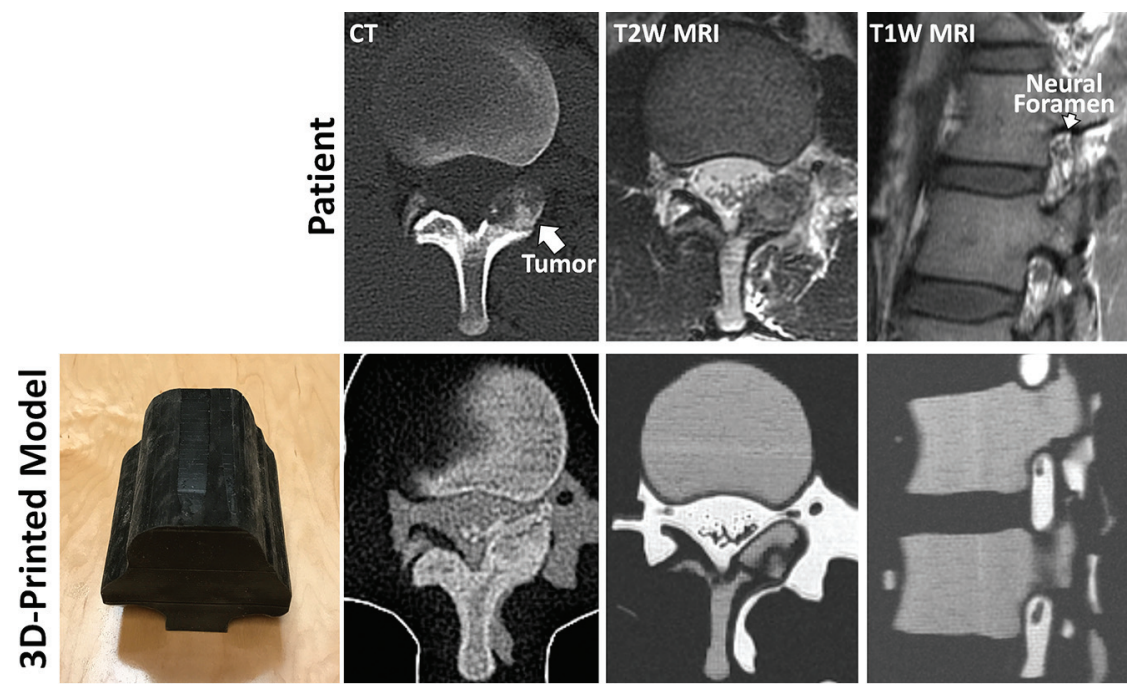

back with the ability to peel away layers of anatomy, providing insight into underlying pathology. To date, 3D-printed simulation of minimally invasive procedures has been limited to conventional angiography procedures, where hollow, radiopaque vascular models can be readily 3D-printed. The next barrier to overcome in this technology is the development of 3D-printing materials and printing techniques that yield models visible with other imaging modalities.

Last year, we began searching for polymeric materials ${ }^{4}$ that possess protons with sufficient mobility to escape substantial dipolar broadening and ultrashort $\mathrm{T} 2$ values, so as to enable imaging of 3Dprinted models in clinical MR imaging systems. ${ }^{5}$ We aimed to leverage such materials to produce models exhibiting different image characteristics for distinct tissues in both $\mathrm{CT}$ and MR imaging.

FIGURE. 3D-printed model of the lumbar spine (bottom left panel) of a patient with L1 left lamina osteoblastoma, designed to replicate the patient's anatomy as seen in the patient's diagnostic CT and MR imaging (top row). The outer black shell of the model is printed in a soft material to mimic soft tissue properties. CT of the 3D-printed model demonstrates cortical and cancellous bone with differential CT Hounsfield units, whereas MR imaging of the model demonstrates cancellous bone with intermediate and cortical bone with dark signal. Neural foramen and nerve root, key structures monitored during MR imaging-guided cryoablation as this patient underwent, are clearly visualized in the MR imaging of the printed model. 
We now bring to your attention this new technique to 3Dprint models replicating patient-specific, complex intertwined anatomy in both CT and MR imaging. A model of a patient with an L1 left lamina osteoblastoma was 3D-printed from the patient's diagnostic CT and MR imaging. Tissues visualized (ie, segmented) in each of the 2 diagnostic scans were spatially aligned to produce a single model combining them. Different mixtures of one MR imaging-visible ${ }^{5}$ and one non-MR imaging-visible material, both of which are radiopaque, were used to produce a model displaying the patient's cortical and cancellous bone and osteoblastoma (as visualized in the patient's CT), spinal nerves, CSF, and epidural fat (as visualized in the patient's MR imaging) with distinct signal characteristics in each imaging modality (Figure).

This model can be used to simulate CT-guided power drilling and subsequent MR imaging-monitored cryoablation (as ice ball formation is not readily visualized on CT in vivo). This is, in fact, the procedure that this patient underwent at our institution. The signal intensity of the model in MR imaging allowed visualization of the neural foramen and nerve root, key structures monitored during this procedure.

Much further research remains in the field, including the development of 3D-printed materials that better match tissue properties and image characteristics (eg, MR relaxation rates and CT Hounsfield units). However, this new 3D-printing technique holds great promise for image-guided procedure planning toward assessing safety and efficacy and also, importantly, for hands-on training where, at present, limited options, such as virtual reality systems, are currently being explored.
Dr. Dimitrios Mitsouras received research funding from the National Institutes of Health-National Institute of Biomedical Imaging and Bioengineering, grant number: K01 EB015868 and Vital Images, a Toshiba Medical Systems Company. Dr. Thomas C. Lee received research funding from the National Institutes of Health-National Institute of Biomedical Imaging and Bioengineering, grant number: U41 RR019703.

\section{REFERENCES}

1. Hinshaw JL, Lubner MG, Ziemlewicz TJ, et al. Percutaneous tumor ablation tools: microwave, radiofrequency, or cryoablation-what should you use and why? Radiographics 2014;34:1344-62 CrossRef Medline

2. Kurup AN, Morris JM, Schmit GD, et al. Neuroanatomic considerations in percutaneous tumor ablation. Radiographics 2013;33:1195215 CrossRef Medline

3. Philip A, Gupta S, Ahrar K, et al. A spectrum of nerve injury after thermal ablation: a report of four cases and review of the literature. Cardiovasc Intervent Radiol 2013;36:1427-35 CrossRef Medline

4. Mitsouras D, Liacouras $P$, Imanzadeh A, et al. Medical 3D printing for the radiologist. Radiographics 2015;35:1965-88 CrossRef Medline

5. Mitsouras D, Lee TC, Liacouras P, et al. Three-dimensional printing of MRI-visible phantoms and MR image-guided therapy simulation. Magn Reson Med 2017;77:613-22 CrossRef Medline

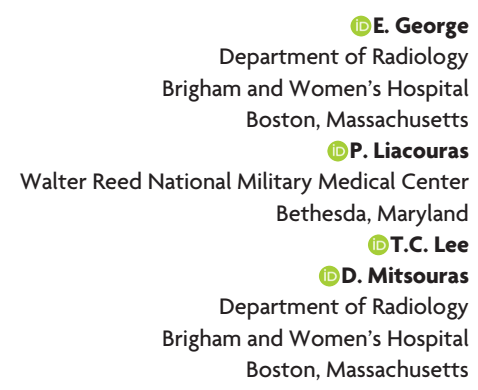

